\title{
Intersubjetividad y diálogo intercultural. La sociología fenomenológica y sus aportes a la comunicación intercultural
}

\author{
Intersubjectivity and Intercultural Dialogue. \\ Phenomenological Sociology and Its Contributions to the \\ Intercultural Communication
}

\author{
Marta Rizo García \\ Universidad Autónoma de la Ciudad de México \\ mrizog@yahoo.com
}

\begin{abstract}
Resumen
La comunicación intercultural se define como cualquier situación comunicativa donde interactúan al menos dos personas procedentes de matrices culturales-geográficas distintas. Algunas definiciones, incluso, ponen énfasis en el momento concreto en que se pone de manifiesto la habilidad para negociar significados culturales en la interacción comunicativa. Por lo tanto, la clave de la comunicación intercultural es la interacción con lo diferente, con todo aquello que objetiva o, sobre todo, subjetivamente, se percibe como distinto, sea cual sea el motivo de distinción (raza, género, clase social, preferencia sexual, etc.). En este texto se reflexiona sobre la interculturalidad desde los conceptos de intersubjetividad y mundo de la vida, procedentes la Sociología Fenomenológica.
\end{abstract}

Palabras clave: intersubjetividad, interculturalidad, comunicación.

\begin{abstract}
Intercultural communication is defined as any communicative situation where at least two people coming from different cultural-geographic matrices interact. Some definitions, even, put emphasis at the concrete moment at which the ability is shown to negotiate meaning cultural in the communicative interaction. Therefore, the key of the intercultural communication is the interaction with different, what the yet objective thing interaction with or, mainly, subjectively, is perceived like different, whatever the reason for distinction (race, sort, social class, sexual preference, etc.). In this text one reflects on the interculturality from the concepts of intersubjectivity and world of life, originating Phenomenological Sociology.
\end{abstract}

Key Words: Intersubjectivity, interculturality, communication. 


\section{La comunicación como puesta en común}

La pregunta por la comunicación puede equipararse con la pregunta por el ser humano. ¿Qué es la comunicación humana? ¿Desde cuándo existe? ¿La humanidad puede comprenderse sin la comunicación? A lo largo de la historia, muchos fenómenos distintos han sido nombrados como "comunicación". Si bien la riqueza de significados puede ser positiva, también puede traer consecuencias negativas como la dispersión y confusión en torno al significado de este concepto. Aunque "comunicación" es un término polisémico, si nos fijamos en el significado etimológico del término obtenemos que comunicación de la voz latina comunicare- significa "poner en común". No es de extrañar, entonces, que a lo largo de la historia la comunicación se haya asociado con conceptos como comunión y comunidad, entre muchos otros.

Luciano Gallino (1995) propone los siguientes modos de concebir a la comunicación, que dan cuenta del carácter polisémico del término: 1) como simple transmisión de un estado o propiedad, que puede referirse a objetos inanimados; 2) como un comportamiento de un ser viviente que influye sobre otro; 3) como intercambio de valores sociales; 4) como transmisión de información; 5) como el acto de compartir significados socialmente intercambiados; y 6) como formación de una unidad social que comparte valores, un determinado modo de vida y un conjunto de reglas. Pese al énfasis dado a las relaciones de intercambio, a la interacción, la investigación en comunicación ha privilegiado la comprensión de la comunicación como transmisión, es decir, se ha estudiado a la comunicación sobre todo en su dimensión mediática, en detrimento de otras formas de comprenderla.

En las siguientes páginas se parte de la comunicación como puesta en común, como interacción, como acción de compartir valores, como modo de establecer vínculos y relaciones entre las personas. La interacción puede ser definida como el intercambio de información entre sujetos sociales. Esta definición general pone el acento en el intercambio, en la relación entre seres humanos con base a la comunicación de información. Por tanto, lo que comúnmente conocemos como comunicación interpersonal se distingue de otros procesos de comunicación por el hecho de no estar mediada mayormente por la tecnología, aunque no lo excluye. En los procesos de interacción que aquí se retoman, los sujetos participan de la interacción en una situación de co-presencia, de presencia física simultánea en el espacio y el tiempo.

En el estudio de la comunicación en el medio social, ésta se halla relacionada con los conceptos de acción e interacción, porque los seres humanos establecen relaciones con los demás por medio de interacciones que pueden calificarse como procesos sociales. En términos generales, la interacción es la acción recíproca entre dos o más agentes. Al margen de quién inicie el proceso de interacción, el resultado es siempre la modificación 
de los estados de los participantes. Los elementos simbólicos, "susceptibles de ser dotados de un significado subjetivo por parte de las personas implicadas en la acción” (Gómez Pellón, 1997: 110), son los que nos permiten hablar de la interacción social. Aunque consideramos que toda interacción requiere de comunicación, se habla también específicamente de la interacción comunicativa, que se comprende como un proceso de organización discursiva entre sujetos que, mediante el lenguaje, actúan en un proceso de constante afectación recíproca.

\section{Intersubjetividad e interacción}

Las aproximaciones a la interacción -y por tanto a la comunicación- son más completas si tomamos en cuenta aportes como los del Interaccionismo simbólico, corriente que se ubica en la Sociología Fenomenológica.

Los principales autores del Interaccionismo Simbólico, corriente deudora de la llamada Escuela de Chicago, fueron Herbert Blumer, George Herbert Mead, Charles Horton Cooley y Erving Goffman. Todos ellos compartieron el interés de analizar a la sociedad en términos de interacciones sociales. Esta corriente destaca la naturaleza simbólica de la vida social, y su finalidad principal fue el estudio de la interpretación por parte de los actores de los símbolos nacidos de sus actividades interactivas. En Symbolic Interaccionism, Herbert Blumer (1968), fundador de la corriente, establece las tres premisas básicas de este enfoque, a saber: 1) Los humanos actúan respecto de las cosas sobre la base de las significaciones que estas cosas tienen para ellos; 2) La significación de estas cosas deriva, o surge, de la interacción social que un individuo tiene con los demás actores; y 3) Estas significaciones se utilizan como un proceso de interpretación efectuado por la persona en su relación con las cosas que encuentra, y se modifican a través de dicho proceso.

Anteriormente se comentó que el Interaccionismo Simbólico puede ubicarse en el marco de la Sociología Fenomenológica, una de las fuentes científicas históricas de la Comunicología que ha sido menos tomada en cuenta en el campo oficial de la comunicación, absolutamente dominado por reflexiones y estudios sobre medios de difusión.

La Sociología Fenomenológica está basada en la filosofía de Husserl (1954) y en el método de comprensión (verstehen) de Max Weber (1977). El debate general gira en torno a cómo se puede lograr el conocimiento, y su aparición debe sustentarse en la comprensión de la fenomenología como instancia de aproximación metodológica a lo cotidiano. Desde un punto de vista epistemológico, la fenomenología implica una ruptura con la formas de pensamiento de la sociología tradicional, ya que enfatiza la necesidad de comprender, más que de explicar, la realidad, sugiriendo que es en el durante, en el aquí y 
en el ahora, donde es posible identificar elementos de significación que describen y construyen lo real. En este sentido, el objetivo general de la fenomenología es describir al hombre en el mundo, no analizarlo o explicarlo. Y para ello, este programa teórico parte de la estructura del contenido y de la interpretación de la realidad, a través de los significados subjetivos que otorgan a ella los sujetos sociales.

Los seres humanos son tratados como sujetos de conciencia, cognoscentes, y no como meros objetos de la naturaleza. El énfasis no se encuentra ni en el sistema social ni en las relaciones funcionales que se dan en la vida en sociedad, sino en la interpretación de los significados del mundo (lebenswelt) y las acciones e interacciones de los sujetos sociales. Del mundo conocido y de las experiencias intersubjetivas compartidas por los sujetos, se obtienen las señales, las indicaciones para interpretar la diversidad de símbolos. Por ello, se dice que el método fenomenológico no parte de una teoría fundada, sino de la observación y descripción del mundo empírico.

Alfred Schütz (1972) es el máximo representante de esta corriente, y su interrogante básica es la siguiente: ¿dónde y cómo se forman los significados de la acción social? Esta pregunta deja entrever que el precedente inmediato de la fenomenología con orientación social lo encontramos en la Escuela de Chicago, concretamente en su interés por conocer y explicar los marcos de referencia de los actores sociales. El centro del programa de Schütz es la cuestión de la sociabilidad como forma superior de intersubjetividad. Esta preocupación básica parte de una idea importante: el estudio de la vida social no puede excluir al sujeto; éste está implicado en la construcción de la realidad objetiva que estudia la ciencia social.

El enfoque de Schütz parte, así entonces, de la necesidad de analizar las relaciones intersubjetivas a partir de las redes de interacción social. En La fenomenología del mundo social (1972), el autor toma como punto de partida para su análisis de la estructura significativa del mundo tanto a la fenomenología de Husserl como a la metodología de Weber (sociología comprensiva). Schütz coincide con Max Weber en el reconocimiento de la importancia de la comprensión del sentido de la acción humana para la explicación de los procesos sociales. Para ambos, la sociedad es un conjunto de personas que actúan en el mundo y cuyas acciones tienen sentido; y es relevante tratar de comprender este sentido para poder explicar los resultados del accionar de los sujetos. Sin embargo, mientras que para Weber la comprensión es el método específico que la sociología utiliza para rastrear los motivos de los actores y así poder asignar sentido a sus acciones, Schütz le otorga a la comprensión un papel mucho más importante: considera que el mundo en el cual vivimos es un mundo de significados, un mundo cuyo sentido y significación es construido por nosotros mismos y los seres humanos que nos precedieron. Por tanto, para Schütz, la comprensión de dichos significados es nuestra manera de vivir en el mundo; la comprensión es ontológica, no sólo metodológica. 
En ambos casos, la propuesta de la Sociología Fenomenológica implica una apuesta por el estudio y explicación del verstehen, es decir, de la experiencia de sentido común en el mundo intersubjetivo de la vida cotidiana. El mundo de la vida cotidiana es, para Schütz,

el ámbito de la realidad en el cual el hombre participa continuamente en formas que son, al mismo tiempo, inevitables y pautadas. El mundo de la vida cotidiana es la región de la realidad en que el hombre puede intervenir y que puede modificar mientras opera en ella mediante su organismo animado (...) sólo dentro de este ámbito podemos ser comprendidos por nuestros semejantes, y sólo en él podemos actuar junto con ellos (1977:25).

La intersubjetividad es la que delinea el campo de la cotidianidad, por un lado, y es el fundamento que posibilita la existencia del mundo de vida, por el otro. Schütz abandona la perspectiva trascendental de Husserl y se centra en la esfera mundana.

\section{Comunicación intercultural, intersubjetividad y mundo de la vida}

La interculturalidad requiere necesariamente de la comunicación. La comunicación, comprendida como interacción, es vínculo entre sujetos, es relación antes que cualquier otra cosa. En la medida en que la comunidad de vida sea mayormente compartida por los sujetos que interactúan, la posibilidad de incrementar la eficacia de la comunicación y en particular de la comunicación intercultural será también mayor, y en consecuencia mayor posibilidad habrá que emisor y receptor entiendan, asuman y aprehendan recíprocamente el sentido que tienen las cosas para cada uno de ellos.

Leer la comunicación intercultural desde los conceptos de intersubjetividad y mundo de la vida, así como leerla desde un enfoque comunicativo centrado en la dimensión de la interacción como contacto entre sistemas de comunicación distintos, permite complejizar las reflexiones en torno a este tema y, sobre todo, permite aterrizar el abordaje de la comunicación intercultural desde la comunicación, y no desde las aproximaciones tradicionales más cercanas a la antropología y la sociología cultural. ¿Qué puede decir la ciencia de la comunicación sobre la comunicación intercultural? ¿Qué aporta la Sociología Fenomenológica -como fuente científica histórica del pensamiento- a la reflexión sobre la comunicación intercultural?

Tomando en cuenta las dimensiones a priori de la Comunicología ${ }^{1}$-a saber, difusión, interacción, estructuración, expresión y observación-, la reflexión sobre la comunicación

\footnotetext{
${ }^{1}$ Para mayor información, revisar textos de Jesús Galindo en el Portal de Comunicología del Grupo hacia una Comunicología Posible (GUCOM), disponible en http:// comunicologia-posible.iespana.es/
} 
intercultural tiene elementos de las cinco dimensiones. La comunicación intercultural se relaciona con la difusión porque en cualquier situación de comunicación intercultural los sujetos intercambian información, saberes construidos desde distintos lugares pero construidos a partir de códigos más o menos compartidos; por otra parte, la comunicación intercultural es fundamentalmente interacción, esto es, relación entre dos sistemas de comunicación -hechos cuerpo en los sujetos- distintos que se vinculan y comparten conocimientos, actitudes, saberes, cosmovisiones, desde lugares distintos y con imágenes de sí mismos y de los otros con quienes interactúan; así mismo, la comunicación intercultural tiene que ver con la dimensión comunicológica de la expresión, comprendida como la comunión resultante del intercambio de signos; por todo lo anterior, y tomando en cuenta que la estructuración refiere a procesos de comunicación en los que están implicadas las categorías de sistemas de información difusión- y sistemas de comunicación -interacción-, la comunicación intercultural es también estructuración.

A continuación, veamos cómo dos de los conceptos básicos de la Sociología Fenomenológica -intersubjetividad y mundo de la vida- permiten una lectura más compleja de la comunicación intercultural. Como se ha dicho anteriormente, para la Sociología Fenomenológica, el individuo es un actor social que reproduce su contexto social a partir de sus interacciones cotidianas. La reflexión se centra en las relaciones intersubjetivas, bajo el ángulo de la interacción, y se otorga un rol relevante a los elementos de negociación y de comunicación en la construcción social de los contextos de sentido. Abordar la Interacción desde la Sociología Fenomenológica implica hablar de la relación entre el yo y el otro, relación básica en toda situación de comunicación y, en este caso, relación fundamental en situaciones de interculturalidad, donde alter -el otro- aparece de forma más clara $\mathrm{o}$, al menos, es determinante para poder considerar que dicha relación es intercultural. Esta relación dialéctica entre el yo y el otro, por tanto, no se inscribe en la reflexión de corte más antropológico de construcción de las identidades y las alteridades, sino que más bien se toma como punto de partida para la construcción social de la realidad.

Como afirma Schütz,

al vivir en el mundo, vivimos con otros y para otros, y orientamos nuestras vidas hacia ellos. $\mathrm{Al}$ vivenciarlos como otros, como contemporáneos y congéneres, como predecesores y sucesores, al unirnos con ellos en la actividad y el trabajo común, influyendo sobre ellos y recibiendo a nuestra vez su influencia, al hacer todas estas cosas, comprendemos la conducta de los otros y suponemos que ellos comprenden la nuestra (1979:39).

El punto de partida de la intersubjetividad, de la consideración de que el otro entiende el mundo de una forma similar a como yo lo comprendo, pudiera ser contradictoria con 
algunas reflexiones en torno a la comunicación intercultural que ponen el acento en el conflicto más que en la negociación, en el choque cultural y en la diferencia, más que en los elementos compartidos. Sin embargo, sea en un marco de conflicto o en un marco de negociación, los sujetos que interactúan son capaces de llegar a consensos -en mayor o menor medida- sobre los significados de la realidad social cotidiana que viven. Y es que el mundo de la cotidianidad es sólo posible si existe un universo simbólico de sentidos compartidos, construidos socialmente, y que permiten la interacción entre subjetividades diferentes. Como afirma Ramon Xirau,

cuando percibo a 'otro' lo percibo como un ser encarnado, como un ser que vive en su cuerpo, es decir, como un ser semejante al mío, que actúa de manera semejante a como actúo y que piensa de manera semejante a la manera en que pienso (Xirau, 2002: 436437).

Así, la Sociología Fenomenológica pone el acento en la semejanza más que en la diferencia, en lo igual más que en lo distinto, en lo común más que en lo distante. Este énfasis ha hecho que la Sociología Fenomenológica haya recibido algunas críticas por no tomar en cuenta, en sus reflexiones, el problema del poder, de la hegemonía, del dominio de unos sobre otros, etc. Si bien la interculturalidad, como cualquier otra situación de comunicación, puede estar mediada por este asunto del poder, no interesa en este artículo poner énfasis en ello, puesto que se parte de la posibilidad de concebir a la comunicación intercultural como una situación que debe ir más allá de la diferencia y el choque, como un lugar en el cual se pueden observar las negociaciones de sentidos, lo compartido, lo consensuado, lo intersubjetivo. Todo ello, como resultado de una comunicación óptima, de una puesta en común que tenga como resultado la comprensión mutua. Como comentamos en un trabajo anterior,

Lo anterior no significa que en situaciones de interculturalidad sólo exista el conflicto y esté ausente la negociación, la aceptación, la coincidencia, el entendimiento. Nada más ajeno a nuestro parecer. La investigación en comunicación intercultural también permite hacer visible los puntos de contacto, las percepciones e ideas convergentes, los puntos de vistas negociables, etc., lo que muchas veces obedece a los modos culturales en que hemos aprendido a interrelacionarnos con el otro, $y$ otras veces a las disposiciones y actitudes de individuos y grupos que, pasando circunstancialmente por alto las disputas históricas por la posesión y la legitimidad social del sentido, pueden sostener relaciones de negociación aunque quizá no de asimilación o aceptación- con ese otro diferente y ajeno (Pech, Rizo, Romeu, 2009: 34-35). 
Como proceso interactivo, la comunicación permite llevar a cabo la interculturalidad, la hace manifiesta, objetivable; y en segundo lugar, como principio de contacto, la comunicación contribuye a la interculturalidad en tanto que puede privilegiar el respeto entre las personas. Por lo anterior, comprender las relaciones interculturales en una situación práctica supone comprender la cultura de los dos mundos en contacto, de dos sistemas simbólicos y cosmovisiones en contacto.

En el ámbito del mundo de la vida, único lugar en el que es posible la comunicación, toda situación de comunicación intercultural se realiza donde hay contacto entre dos o más entramados de significados y sentidos; y yendo más allá, es posible hablar de interculturalidad sólo cuando un sujeto comienza a entender, en el sentido de asumir, el significado y el valor de las cosas y objetos para el otro. Para ello, el marco de sentido proporcionado por la intersubjetividad -requisito indispensable para la existencia del mundo de la vida- se convierte en donador básico de esta capacidad de los seres humanos para asumir y comprender los significados tal y como los otros los asumen. $\mathrm{O}$ dicho de otra forma,

este marco de referencia intersubjetivo se construye mediante la praxis, y se instaura como un sistema socio-cognitivo desde donde la comunicación, y particularmente la comunicación intercultural, es posible. De esa manera, la intersubjetividad queda anclada a través de los sistemas simbólicos (Pech, Rizo y Romeu, 2009: 48).

A modo de síntesis, se puede afirmar que los participantes en un encuentro intercultural interactúan apoyándose en suposiciones culturales propias, que funcionan como pantallas perceptuales de los mensajes que intercambian, es decir, como modos de ver y comprender el mundo. Estas "pantallas" serían lo que el propio Schütz denomina situación biográfica y acervo de conocimiento. La primera remite al comportamiento específico de cada individuo, es decir, a que cada autor lleva una secuencia en su vida de las interpretaciones de lo que encuentra en el mundo, según la perspectiva de sus intereses, motivaciones e ideología. Por ello, aunque la realidad del sentido común es dada a los sujetos en formas culturales o históricas de validez universal, la forma en que éstas se expresan en las vidas individuales depende de la totalidad de experiencias que una persona construye en el curso de su existencia concreta. Así entonces, la situación biográfica condiciona el modo de actuar de los sujetos. Con respecto al acervo de conocimiento, también conocido como repositorio de conocimiento disponible, éste hace referencia a aquello de lo que disponen los sujetos, y está integrado por tipificaciones del mundo del sentido común. El mundo de la vida incluye además de seres vivos y objetos inertes, seres y objetos que son percibidos típicamente y que le son familiares al sujeto. A lo largo de su vida, el sujeto incorpora y acumula una gran cantidad de indicaciones, que luego usará 
como técnicas para comprender o al menos controlar aspectos de su experiencia cotidiana.

El éxito o fracaso de la interacción dependerá, fundamentalmente, de la familiaridad de los participantes con los antecedentes de su interlocutor, es decir, del grado de conocimiento de uno con respecto al otro, las percepciones de las diferencias que los separan y la reciprocidad del propósito de la comunicación. Sólo así, la comunicación pasará de ser un acto individual con ejecutantes individuales a ser una experiencia "compartida".

\section{Reflexiones finales}

La comunicación intercultural parte de la dimensión interactiva y relacional de la comunicación. Interactiva porque concibe al proceso comunicativo como la puesta en escena de relaciones en acción; relacional porque el peso de cada una de esas relaciones condiciona constantemente la dirección y el sentido de dicha interacción. Pese a ello, no puede dejarse de lado completamente que la comunicación intercultural es una comunicación conflictiva, pues desencadena interacciones que no siempre están ceñidas a la simetría y la equidad. Desigualdades y asimetrías que obedecen a las condiciones históricas concretas que enmarcan la interacción, mismas que, por una parte dan muestra de las relaciones de dominación entre los diferentes grupos en conflicto, y por la otra, permiten descubrir las densidades y texturas que las constituyen. Estos asuntos, sin embargo, no fueron objeto de reflexión por parte de Alfred Schütz y su propuesta sociofenomenológica, corriente que se ha tomado como marco de referencia en este artículo.

En cualquier situación de interacción intercultural, dos o más sujetos establecen un contacto e interactúan desde sus situaciones biográficas concretas y llevando consigo sus repertorios de conocimiento disponibles. Este contacto produce un espacio en el cual se negocian las interpretaciones del mundo, para lo cual es fundamental que los sujetos posean predisposiciones y competencias hacia el contacto con lo otro, con lo distinto. La relación de negociación y/o conflicto está marcada por las distintas representaciones del mundo, así como por la existencia de la zona porosa de relación que hemos denominado frontera interna. Esta última se comprende como

un lugar simbólico donde simultánea y dialécticamente se producen y reproducen los significados con los que el sujeto se percibe a sí mismo y a los otros, y por medio de los cuales aprehende el mundo que le rodea ( ... no está circunscrita a un límite o perímetro territorial, sino más bien a un lugar amorfo, en el que se activan los universos simbólicos de los sujetos y grupos. Se trata de una zona en la que se segmentan, se distinguen y se separan identidades, representaciones, significados, cosmovisiones, al tiempo que se mezclan, se 
yuxtaponen, se negocian y se comparten (Pech, Rizo y Romeu, 2009: 36-37).

El siguiente mapa integra los conceptos mencionados hasta el momento y esquematiza los términos básicos que permiten pensar la comunicación intercultural desde un enfoque comunicológico que privilegia la concepción socio-fenomenológica de la comunicación.

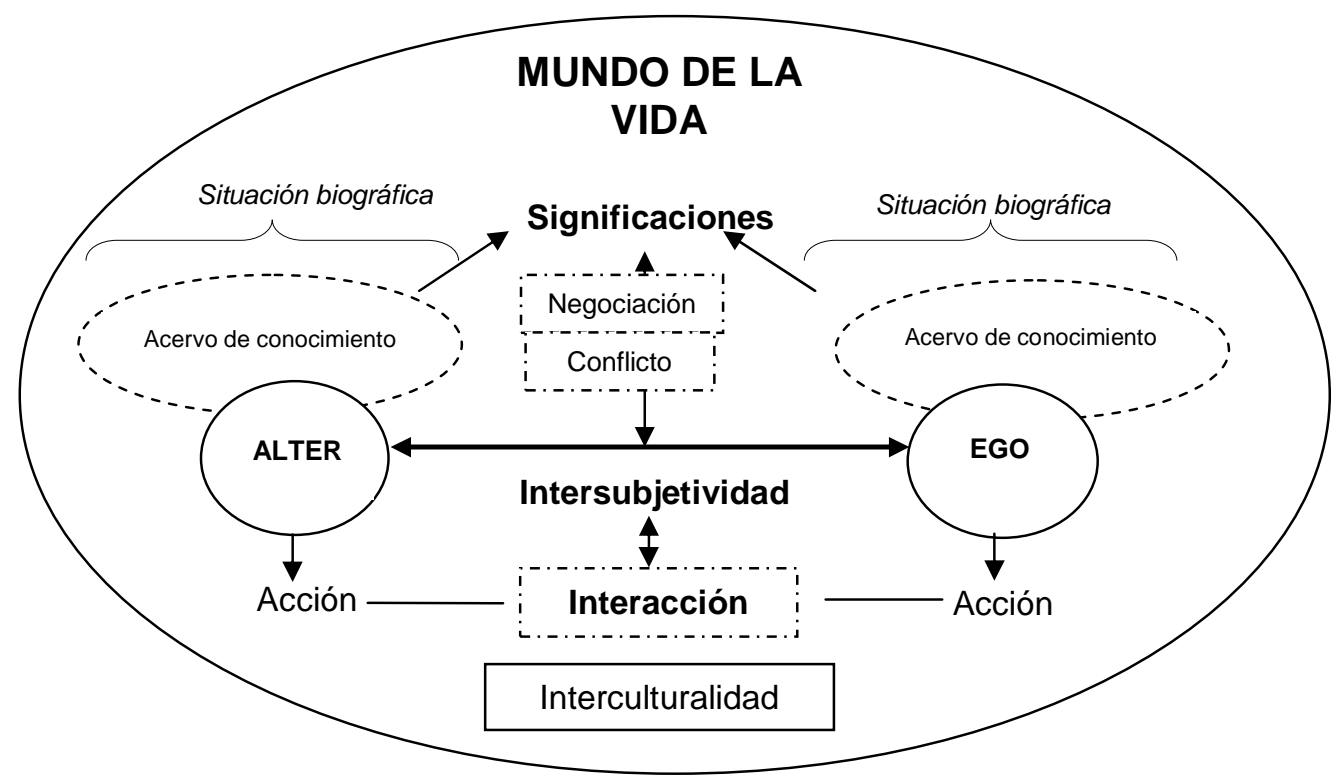

Figura 1. Mapa conceptual. (fuente: elaboración propia.)

La relación entre sujetos -que actúan e interactúan- se produce en el marco del mundo de la vida cotidiana, un mundo intersubjetivo. La interacción entre alter y ego da lugar a significaciones -con un mayor o menor grado de consenso, es decir, en un marco donde prime la negociación o donde prime el conflicto- sobre el mundo. Estas significaciones están originadas por los acervos de conocimiento disponibles que cada sujeto lleva consigo -producto de su situación biográfica- a la situación de interacción intercultural en la que participa cotidianamente.

\section{Referencias bibliográficas}

Blumer, Herbert (1968). Symbolic Interactionism. Perspective and Method. New Jersey: Prentice HallEnglewood Cliffs. 
Galindo, Jesús (2003). "Notas para una comunicología posible. Elementos para una matriz y un programa de configuración conceptual-teórica”. Obtenido el 1 de febrero de 2010 en http://comunicologia-posible.iespana.es/principal/textos\%20gucom\%20y\%20redecom.html

Galindo, Jesús (2004). "Apuntes de historia de una comunicología posible. Hipótesis de configuración y trayectoria”. En: Comunicología: indicios y conjeturas, Departamento de Comunicación de la Universidad Iberoamericana de México, Núm. 1, Primavera 2004. Obtenido el 1 de febrero de 2010 de http://revistacomunicologia.org/index.php?option=com_content\&task=view\&id=36\&Itemi $\mathrm{d}=97$

Gómez Pellón, Eloy (1997). “Lo evolución del concepto de etnografia”, en Aguirre Baztán, Ángel (editor) Etnografía. Metodología cualitativa en la investigación sociocultural. México: Alfaomega.

Husserl, Edmund (1954). Ideas relativas a una fenomenología pura y una filosofía fenomenológica. México: Fondo de Cultura Económica.

Pech, Cynthia; Rizo, Marta; Romeu, Vivian (2009). "El habitus y la intersubjetividad como conceptos clave para la comprensión de las fronteras internas. Un acercamiento desde las propuestas de Bourdieu y Schütz", en revista Frontera Norte, Vol. 21, Núm. 41, Enero-Junio de 2009, El Colegio de la Frontera Norte, Tijuana (México), pp. 33-52. Obtenido el 1 de febrero de 2010 en http://aplicaciones.colef.mx:8080/fronteranorte/articulos/FN41/2-f41.pdf

Rizo, Marta; Romeu, Vivian (2006). "Cultura y comunicación intercultural. Aproximaciones conceptuales", en dossier "Comunicação e estudos culturais" de E-Compos, Revista de la Associação Nacional dos Programas de Pós-Graduação em Comunicação-Compôs, Brasil. Octubre de 2006. Obtenido el 1 de febrero de 2010 http://www.compos.org.br/seer/index.php/e-compos/article/viewArticle/85

Schütz, Alfred (1972). El problema de la realidad social. Buenos Aires: Amorrortu.

Schütz, Alfred (1977). La construcción significativa del mundo social. Introducción a la sociología comprensiva. Barcelona: Paidós.

Schütz, Alfred (1979). Estudios sobre teoría social. Buenos Aires: Amorrortu editores.

Weber, Max (1977). Ensayos de metodología sociológica. Buenos Aires: Amorrortu editores.

Xirau, Ramón (2002). Introducción a la historia de la filosofía. México: UNAM. 